Korean J. Math. 21 (2013), No. 4, pp. 421-428

http://dx.doi.org/10.11568/kjm.2013.21.4.421

\title{
ON COEFFICIENTS OF NILPOTENT POLYNOMIALS IN SKEW POLYNOMIAL RINGS
}

\author{
SAng Bok NAm, Sung Ju RYu*, AND SANG Jo Yun
}

\begin{abstract}
We observe the basic structure of the products of coefficients of nilpotent (left) polynomials in skew polynomial rings. This study consists of a process to extend a well-known result for semi-Armendariz rings. We introduce the concept of $\alpha$-skew n-semiArmendariz ring, where $\alpha$ is a ring endomorphism. We prove that a ring $R$ is $\alpha$-rigid if and only if the $n$ by $n$ upper triangular matrix ring over $R$ is $\bar{\alpha}$-skew $n$-semi-Armendariz. This result are applicable to several known results.
\end{abstract}

\section{Introduction}

Throughout this paper all rings are associative with identity unless otherwise stated. A ring is called reduced if it has no nonzero nilpotent elements. Let $\alpha$ be an endomorphism of a ring $R$. A skew polynomial ring with an indeterminate $x$ over $R$, written by $R[x ; \alpha]$, means the polynomial ring $R[x]$ with a new multiplication $x r=\alpha(r) x$ for $r \in R$. In this situation each element of $R[x ; \alpha]$ is called (left) polynomial.

An endomorphism $\alpha$ is called rigid by Krempa [10] when $a \alpha(a)=0$ implies $a=0$ for $a \in R$. It is trivial that rigid endomorphisms are

Received October 18, 2013. Revised November 25, 2013. Accepted November 25, 2013.

2010 Mathematics Subject Classification: 16S36, 16S50.

Key words and phrases: $\alpha$-skew $n$-semi-Armendariz, $\alpha$-rigid ring, skew polynomial ring, matrix ring, reduced ring.

*Corresponding author.

(C) The Kangwon-Kyungki Mathematical Society, 2013.

This is an Open Access article distributed under the terms of the Creative commons Attribution Non-Commercial License (http://creativecommons.org/licenses/by -nc/3.0/) which permits unrestricted non-commercial use, distribution and reproduction in any medium, provided the original work is properly cited. 
injective. Hong et al. [6] called a ring $\alpha$-rigid if it has a rigid endomorphism $\alpha$ of $R$ and they showed that $\alpha$-rigid rings are reduced and $\alpha$ is a monomorphism.

For a reduced ring $R$ Armendariz [3, Lemma 1] proved that $a_{i} b_{j}=0$ for all $i, j$ whenever $f(x) g(x)=0$ where $f(x)=\sum_{i=0}^{m} a_{i} x^{i}, g(x)=$ $\sum_{j=0}^{n} b_{j} x^{j}$ are in $R[x] \ldots\left(^{*}\right)$. Rege et al. [13] called a ring (not necessarily reduced) Armendariz if it satisfies (*). Reduced rings are Armendariz by [3, Lemma 1]. The structure of Armendariz rings was observed by many authors containing Anderson et al. [1], Hirano [4], Huh et al. [7], Kim et al. [9], Lee et al. [12], Rege et al. [13], etc. Due to Hong et al. [5], a ring $R$ is called a skew Armendariz ring with an endomorphism $\alpha$ (or simply an $\alpha$-skew Armendariz ring) provided that for $p=\sum_{i=0}^{m} a_{i} x^{i}, q=$ $\sum_{j=0}^{n} b_{j} x^{j} \in R[x ; \alpha], p q=0$ implies $a_{i} \alpha^{i}\left(b_{j}\right)=0$ for all $i, j$. Every $\alpha$ rigid ring is $\alpha$-skew Armendariz by [5, Corollary 4]. Jeon et al. [8] called a ring $n$-semi-Armendariz provided that if $f(x)=a_{0}+a_{1} x+\cdots+a_{m} x^{m}$ in $R[x]$ satisfies $f(x)^{n}=0$ then $a_{i_{1}} a_{i_{2}} \cdots a_{i_{n}}=0$ for any choice of $a_{i_{j}}$ 's in $\left\{a_{0}, \cdots, a_{m}\right\}$ where $j=1, \ldots, n$ (of course $n \geq 2$ ). A ring is called semi-Armendariz if it is $n$-semi-Armendariz for all $n \geq 2$. Armendariz rings are semi-Armendariz by [2, Proposition 1], but the converse need not hold since the 2 by 2 upper triangular matrix ring over a reduced ring is semi-Armendariz by [8, Theorem 1.2].

In the following we extend the concept of semi-Armendariz rings to skew polynomial rings. One can see details related to semi-Armendariz rings in [8]. In this note we will call a ring $R \alpha$-skew $n$-semi-Armendariz provided that $f(x)=a_{0}+a_{1} x+\cdots+a_{m} x^{m}$ in $R[x ; \alpha]$ satisfies $f(x)^{n}=0$ then

$$
a_{i_{1}} \alpha^{i_{1}}\left(a_{i_{2}}\right) \cdots \alpha^{i_{1}+\cdots+i_{n-1}}\left(a_{i_{n}}\right)=0
$$

for any choice of $a_{i_{j}}$ 's in $\left\{a_{0}, \cdots, a_{m}\right\}$ where $j=1, \ldots, n$ (of course $n \geq 2$ ). A ring is called $\alpha$-skew semi-Armendariz if it is $\alpha$-skew $n$-semiArmendariz for all $n \geq 2$. Every $\alpha$-skew Armendariz ring is $\alpha$-skew semi-Armendariz by Lemma 2(4) to follow, but the converse need not hold by the following example.

Let $R$ be a ring and $n$ be a positive integer. Let $\operatorname{Mat}_{n}(R)$ denote the $n$ by $n$ matrix ring over $R$ and $I_{n}$ be the identity of $\operatorname{Mat}_{n}(R)$. We use $U_{n}(R)$ (resp. $L_{n}(R)$ ) to denote the $n$ by $n$ upper (resp. lower) triangular matrix ring over $R$. $E_{i j}$ denotes the $n$ by $n$ matrix with $(i, j)$-entry 1 and zero elsewhere. Let $\alpha$ be an endomorphism of a ring $R$. We define an endomorphism $\bar{\alpha}$ of any subring in $\operatorname{Mat}_{n}(R)$ by $\left(a_{i j}\right) \mapsto\left(\alpha\left(a_{i j}\right)\right)$. 
EXAMPLE 1. Let $\mathbb{Q}(t)$ be the quotient field with an indeterminate $t$ over $\mathbb{Q}$ and put $R=\mathbb{Q}(t)$. Define $\alpha: R \rightarrow R$ by $\frac{f(t)}{g(t)} \mapsto \frac{f\left(t^{2}\right)}{g\left(t^{2}\right)}$ then $R$ is $\alpha$-rigid and $\alpha$ is a monomorphism of $R$ with $\alpha(1)=1$. $U_{2}(R)$ is $\bar{\alpha}$-skew semi-Armendariz by Theorem 4 to follow. For $p=$ $\left(\begin{array}{ll}1 & 0 \\ 0 & 0\end{array}\right)+\left(\begin{array}{ll}1 & 1 \\ 0 & 0\end{array}\right) x, q=\left(\begin{array}{cc}0 & 0 \\ 0 & -1\end{array}\right)+\left(\begin{array}{cc}0 & 1 \\ 0 & -1\end{array}\right) x \in U_{2}[x ; \bar{\alpha}]$, we have $p q=0$ but $\left(\begin{array}{ll}1 & 1 \\ 0 & 0\end{array}\right) \bar{\alpha}\left(\left(\begin{array}{cc}0 & 0 \\ 0 & -1\end{array}\right)\right) \neq 0$. Thus $U_{2}(R)$ is not $\bar{\alpha}$-skew Armendariz.

\section{Lemmas}

Due to Lambek [11], a ring $R$ is called symmetric if $r s t=0$ implies $r t s=0$ for all $r, s, t \in R$. Lambek proved that a ring $R$ is symmetric if and only if $r_{1} r_{2} \cdots r_{n}=0$ implies $r_{\sigma(1)} r_{\sigma(2)} \cdots r_{\sigma(n)}=0$ for any permutation $\sigma$ of the set $\{1,2, \ldots, n\}$, where $n \geq 1$ and $r_{i} \in R$ for all $i$ (see [11, Proposition 1]). This result was independently shown by Anderson and Camillo in [2, Theorem I.3]. We use this fact without mentioning.

Lemma 2. (1) Let $R$ be a reduced ring, $n$ be any positive integer and $r_{i} \in R$ for $i=1, \ldots, n$. Then $r_{1} r_{2} \cdots r_{n}=0$ implies $r_{\sigma(1)} R r_{\sigma(2)} R \cdots$ $R r_{\sigma(n)}=0$ for any permutation $\sigma$ of the set $\{1,2, \ldots, n\}$.

(2) Let $R$ be an $\alpha$-rigid ring and $a_{i} \in R$ for $i=1, \ldots, m$. If $a_{1} \cdots a_{m}=$ 0 then $\alpha^{n_{1}}\left(a_{1}\right) \cdots \alpha^{n_{m}}\left(a_{m}\right)=0$ for any positive integers $n_{i}$ 's.

(3) $A$ ring $R$ is $\alpha$-rigid if and only if $\alpha^{k_{1}}\left(a_{1}\right) \cdots \alpha^{k_{m}}\left(a_{m}\right)=0$ (for some positive integers $k_{i}$ 's) implies $a_{1} \cdots a_{m}=0$ and $R$ is reduced and $\alpha$ is a monomorphism, where $a_{i} \in R$ for all $i$.

(4) $A$ ring $R$ is $\alpha$-skew Armendariz if and only if $f_{1} \cdots f_{n}=0$ implies $a_{1_{j}} \alpha^{1_{j}}\left(a_{2_{j}}\right) \cdots \alpha^{1_{j}+2_{j}+\cdots+(n-1)_{j}}\left(a_{n_{j}}\right)=0$, where $f_{1}, \ldots, f_{n} \in R[x ; \alpha]$ and $a_{i_{j}} x^{i_{j}}$ is any term of $f_{i}$ with $a_{i_{j}} \in R$.

Proof. (1) It is easily checked that reduced rings are symmetric. Thus we obtain the result.

(2) From [6, Lemma 4(i)], it is true.

(3) By $(2), \alpha^{k_{1}}\left(a_{1}\right) \cdots \alpha^{k_{m}}\left(a_{m}\right)=0$ implies $\alpha^{M}\left(a_{1}\right) \cdots \alpha^{M}\left(a_{m}\right)=$ $\alpha^{M}\left(a_{1} \cdots a_{m}\right)=0$ where $M=\max \left\{k_{1}, \ldots, k_{m}\right\}$. Thus $a_{1} \cdots a_{m}=0$, since $\alpha$ is a monomorphism. 
For the converse, let $r \alpha(r)=0$ for $r \in R$. Then $\alpha(r) \alpha^{2}(r)=0$ and hence $r^{2}=0$, since $\alpha$ is a monomorphism. Since $R$ is reduced, $r=0$.

(4) It suffices to show the necessity. We first compute the case of $n=3$. Let $R$ be an $\alpha$-skew Armendariz ring and suppose that $f_{1} f_{2} f_{3}=0$ for $f_{1}, f_{2}, f_{3} \in R[x ; \alpha]$. We also use $\alpha$ for the endomorphism of $R[x ; \alpha]$ defined by $\sum a_{i} x^{i} \mapsto \sum \alpha\left(a_{i}\right) x^{i}$. Then $0=a_{1_{j}} \alpha^{1_{j}}\left(f_{2} f_{3}\right)=$ $\left(\sum a_{1_{j}} \alpha^{1_{j}}\left(a_{2_{j}}\right) x^{2_{j}}\right) \alpha^{1_{j}}\left(f_{3}\right)$ and so $a_{1_{j}} \alpha^{1_{j}}\left(a_{2_{j}}\right) \alpha^{1_{j}+2_{j}}\left(a_{3_{j}}\right)=0$, where $f_{2}=$ $\sum_{2_{j}} a_{2_{j}} x^{2_{j}}$ and $f_{3}=\sum_{3_{j}} a_{3_{j}} x^{3_{j}}$.

Therefore we can inductively obtain $a_{1_{j}} \alpha^{1_{j}}\left(a_{2_{j}}\right) \cdots \alpha^{1_{j}+2_{j}+\cdots+(n-1)_{j}}$ $\left(a_{n_{j}}\right)=0$ for $n \geq 4$, where $f_{k}=\sum_{k_{j}} a_{k_{j}} x^{k_{j}}$ for $k=1, \ldots, n$.

The following is obtained naturally by definition.

Lemma 3. (1) The class of $\alpha$-skew ( $n$-semi-)Armendariz rings is closed under subrings.

(2) Any direct product of $\alpha$-skew $n$-semi-Armendariz rings is $\alpha$-skew $n$-semi-Armendariz.

(3) Any direct sum of $\alpha$-skew $n$-semi-Armendariz rings is $\alpha$-skew $n$ semi-Armendariz.

\section{Main Theorem}

For a ring $R$ and a positive integer $n$ define

$$
N_{n}(R)=\left\{A \in U_{n}(R) \mid \text { each diagonal entry of } A \text { is zero }\right\} .
$$

Theorem 4. Let $R$ be a ring, $\alpha$ be a monomorphism of $R$ with $\alpha(1)=1$, and $n$ be a positive integer. Then the following conditions are equivalent:

(1) $R$ is $\alpha$-rigid;

(2) $U_{h}(R)$ is $\bar{\alpha}$-skew $n$-semi-Armendariz for $h=1,2, \cdots, n+1$;

(3) $U_{n}(R)$ is $\bar{\alpha}$-skew $n$-semi-Armendariz;

(4) $L_{h}(R)$ is $\bar{\alpha}$-skew $n$-semi-Armendariz for $h=1,2, \cdots, n+1$;

(5) $L_{n}(R)$ is $\bar{\alpha}$-skew $n$-semi-Armendariz.

Proof. We extend the proof of [8, Theorem 1.2] to this situation. $(1) \Rightarrow(2)$ : Suppose that $R$ is $\alpha$-rigid. Then $R$ is reduced. It suffices to prove that $U_{n+1}(R)$ is $\bar{\alpha}$-skew $n$-semi-Armendariz by Lemma $3(1)$. 
Let $f(x)=A_{0}+A_{1} x+\cdots+A_{m} x^{m} \in U_{n+1}(R)[x ; \bar{\alpha}]$ with $f(x)^{n}=0$ $(n \geq 2)$. Write

$$
A_{i}=\left(a(i)_{u v}\right) \text { for } i=0,1, \ldots, m \text { with } a(i)_{u v}=0 \text { for } u>v .
$$

We will use the $\alpha$-rigidness and reducedness of $R$ without referring. From $f(x)^{n}=0$, we have the system of equations

$$
\sum_{s_{1}+s_{2}+\cdots+s_{n}=k} A_{s_{1}} \bar{\alpha}^{s_{1}}\left(A_{s_{2}}\right) \cdots \bar{\alpha}^{\sum_{t=1}^{n-1} s_{t}}\left(A_{s_{n}}\right)=0 \text { for } k=0,1, \ldots, m n .
$$

From $A_{0}^{n}=0$, we have $a(0)_{11}=\cdots=a(0)_{(n+1)(n+1)}=0$. From $A_{m} \bar{\alpha}^{m}\left(A_{m}\right) \cdots \bar{\alpha}^{(n-1) m}\left(A_{m}\right)=0$, we have $a(m)_{i i} \alpha^{m}\left(a(m)_{i i}\right) \cdots \alpha^{(n-1) m}$ $\left(a(m)_{i i}\right)=0$ for $i=1, \ldots, n+1$; hence we get $a(m)_{i i}^{n}=0$ by Lemma $2(3)$, entailing that $a(m)_{i i}=0$. Thus $A_{0}, A_{m} \in N_{n+1}(R)$.

Consider the coefficient of $f(x)^{n}$ of degree $n$. In the equality

$$
\sum_{s_{1}+\cdots+s_{n}=n} A_{s_{1}} \bar{\alpha}^{s_{1}}\left(A_{s_{2}}\right) \cdots \bar{\alpha}^{\sum_{t=1}^{n-1} s_{t}}\left(A_{s_{n}}\right)=0
$$

any term (except $\left.A_{1} \bar{\alpha}\left(A_{1}\right) \cdots \bar{\alpha}^{(n-1)}\left(A_{1}\right)\right)$ contains $\bar{\alpha}^{s}\left(A_{0}\right)$ (for some $s$ ) as a factor, and so it is contained in $N_{n+1}(R)$ from $A_{0} \in N_{n+1}(R)$. Consequently $A_{1} \bar{\alpha}\left(A_{1}\right) \cdots \bar{\alpha}^{(n-1)}\left(A_{1}\right) \in N_{n+1}(R)$, and so we get $A_{1} \in$ $N_{n+1}(R)$ by the same computation as $A_{m}$.

Next we proceed by induction on $i=0,1, \ldots, m-1$. Consider the coefficient of $f(x)^{n}$ of degree $n i$. In the equality

$$
\sum_{s_{1}+\cdots+s_{n}=n i} A_{s_{1}} \bar{\alpha}^{s_{1}}\left(A_{s_{2}}\right) \cdots \bar{\alpha}^{\sum_{t=1}^{n-1} s_{t}}\left(A_{s_{n}}\right)=0,
$$

any term (except $A_{i} \bar{\alpha}\left(A_{i}\right) \cdots \bar{\alpha}^{(n-1)}\left(A_{i}\right)$ ) contains $\bar{\alpha}^{s}\left(A_{j}\right)$ (for some $s$ ) with $j<i$ as a factor, and so it is contained in $N_{n+1}(R)$ by induction hypothesis. Consequently $A_{i} \bar{\alpha}\left(A_{i}\right) \cdots \bar{\alpha}^{(n-1)}\left(A_{i}\right) \in N_{n+1}(R)$ and then $A_{i} \in N_{n+1}(R)$ by the same computation as $A_{m}$. Whence we have

$$
a(i)_{11}=a(i)_{22}=\cdots=a(i)_{(n+1)(n+1)}=0
$$

for $i=0,1, \ldots, m$ and it follows that

$$
\begin{array}{r}
A_{s_{1}} \bar{\alpha}^{s_{1}}\left(A_{s_{2}}\right) \cdots \bar{\alpha}^{\sum_{t=1}^{n-1} s_{t}}\left(A_{s_{n}}\right)=\left(a\left(s_{1}\right)_{12} \alpha^{s_{1}}\left(a\left(s_{2}\right)_{23}\right)\right. \\
\left.\cdots \alpha^{\sum_{t=1}^{n-1} s_{t}}\left(a\left(s_{n}\right)_{n(n+1)}\right)\right) E_{1(n+1)}
\end{array}
$$


for any choice of $s_{i}$ 's. But this equality is equivalent to the system of equations

(*)

$$
\sum_{s_{1}+s_{2}+\cdots+s_{n}=k} a\left(s_{1}\right)_{12} \alpha^{s_{1}}\left(a\left(s_{2}\right)_{23}\right) \cdots \alpha^{\sum_{t=1}^{n-1} s_{t}}\left(a\left(s_{n}\right)_{n(n+1)}\right)=0
$$

for $k=0,1, \ldots, m n$. For the case of $k=1$, if we multiply the equation

$$
\sum_{s_{1}+s_{2}+\cdots+s_{n}=1} a\left(s_{1}\right)_{12} \alpha^{s_{1}}\left(a\left(s_{2}\right)_{23}\right) \cdots \alpha^{\sum_{t=1}^{n-1} s_{t}}\left(a\left(s_{n}\right)_{n(n+1)}\right)=0
$$

on the right side by $a(0)_{12} \cdots a(0)_{(i-1) i} a(0)_{(i+1)(i+2)} \cdots a(0)_{n(n+1)}$, then from $a(0)_{12} \cdots a(0)_{n(n+1)}=0$ and Lemma 2(1) we obtain

$$
\begin{array}{r}
\left(a(0)_{12} \cdots a(0)_{(i-1) i} a(1)_{i(i+1)} \alpha\left(a(0)_{(i+1)(i+2)}\right) \cdots \alpha\left(a(0)_{n(n+1)}\right)\right) \\
\left(a(0)_{12} \cdots a(0)_{(i-1) i} a(0)_{(i+1)(i+2)} \cdots a(0)_{n(n+1)}\right)=0
\end{array}
$$

for $i=1, \ldots, n$ since every other term contains $a(0)_{i(i+1)}$ for $i=1,2, \ldots, n$ as factors. It then follows that

$$
\left(a(0)_{12} \cdots a(0)_{(i-1) i} a(1)_{i(i+1)} \alpha\left(a(0)_{(i+1)(i+2)}\right) \cdots \alpha\left(a(0)_{n(n+1)}\right)\right)^{2}=0
$$

by Lemma $2(1,2)$, and so

$$
a(0)_{12} \cdots a(0)_{(i-1) i} a(1)_{i(i+1)} \alpha\left(a(0)_{(i+1)(i+2)}\right) \cdots \alpha\left(a(0)_{n(n+1)}\right)=0 .
$$

We proceed by induction on $k=0,1, \ldots, m n-1$. Let $v$ be maximal in the set $\left\{s_{i} \mid s_{1}+s_{2}+\cdots+s_{n}=k\right\}$ where $k \in\{1, \ldots, m n-1\}$. Consider a term

$$
a\left(s_{1}\right)_{12} \alpha^{s_{1}}\left(a\left(s_{2}\right)_{23}\right) \cdots \alpha^{\sum_{t=1}^{n-1} s_{t}}\left(a\left(s_{n}\right)_{n(n+1)}\right)
$$

with $s_{i}=v$ and $s_{1}+s_{2}+\cdots+s_{n}=k$. Note that not all $s_{j}$ 's are equal by the choice of $v$. Multiplying $\sum_{s_{1}+s_{2}+\cdots+s_{n}=k} a\left(s_{1}\right)_{12} \alpha^{s_{1}}\left(a\left(s_{2}\right)_{23}\right) \cdots$ $\alpha^{\sum_{t=1}^{n-1} s_{t}}\left(a\left(s_{n}\right)_{n(n+1)}\right)=0$ on the right side by

$$
\begin{array}{r}
a\left(s_{1}\right)_{12} \cdots \alpha^{\sum_{t=1}^{i-2} s_{t}}\left(a\left(s_{i-1}\right)_{(i-1) i}\right) \alpha^{\sum_{t=1}^{i} s_{t}}\left(a\left(s_{i+1}\right)_{(i+1)(i+2)}\right) \\
\cdots \alpha^{\sum_{t=1}^{n-1} s_{t}}\left(a\left(s_{n}\right)_{n(n+1)}\right),
\end{array}
$$

we have

$$
\begin{aligned}
& \left(a\left(s_{1}\right)_{12} \alpha^{s_{1}}\left(a\left(s_{2}\right)_{23}\right) \cdots \alpha^{\sum_{t=1}^{n-1} s_{t}}\left(a\left(s_{n}\right)_{n(n+1)}\right)\right) \\
& \left(a\left(s_{1}\right)_{12} \cdots \alpha^{\sum_{t=1}^{i-2} s_{t}}\left(a\left(s_{i-1}\right)_{(i-1) i}\right)\right. \\
& \left.\alpha^{\sum_{t=1}^{i} s_{t}}\left(a\left(s_{i+1}\right)_{(i+1)(i+2)}\right) \cdots \alpha^{\sum_{t=1}^{n-1} s_{t}}\left(a\left(s_{n}\right)_{n(n+1)}\right)\right)=0
\end{aligned}
$$


by induction hypothesis and Lemma $2(1,2)$ since every other term (after multiplying) contains

$$
\alpha^{h_{1}}\left(a\left(t_{1}\right)_{12}\right), \cdots, \alpha^{h_{n}}\left(a\left(t_{n}\right)_{n(n+1)}\right)
$$

(for some $h_{i}$ 's), with $t_{1}+\cdots+t_{n} \leq k-1$, as factors. Thus we have

$$
\left(a\left(s_{1}\right)_{12} \alpha^{s_{1}}\left(a\left(s_{2}\right)_{23}\right) \cdots \alpha^{\sum_{t=1}^{n-1} s_{t}}\left(a\left(s_{n}\right)_{n(n+1)}\right)\right)^{2}=0
$$

by Lemma $2(1,2)$, entailing $a\left(s_{1}\right)_{12} \alpha^{s_{1}}\left(a\left(s_{2}\right)_{23}\right) \cdots \alpha^{\sum_{t=1}^{n-1} s_{t}}\left(a\left(s_{n}\right)_{n(n+1)}\right)=$ 0 . Next take such $v$ in the remaining terms and apply the same computation method.

Proceeding in this manner we finally get to $a\left(u_{1}\right)_{12} \alpha^{u_{1}}\left(a\left(u_{2}\right)_{23}\right) \cdots$ $\alpha^{\sum_{t=1}^{n-1} u_{t}}\left(a\left(u_{n}\right)_{n(n+1)}\right)=0$ for any choice of $u_{i}$ 's such that $u_{1}+u_{2}+\cdots+$ $u_{n}=k$ and not all $u_{i}^{\prime}$ 's are equal. In this situation, if $k$ is divisible by $n$ then we finally have $a\left(\frac{k}{n}\right)_{12} \alpha^{\frac{k}{n}}\left(a\left(\frac{k}{n}\right)_{23}\right) \cdots \alpha^{\frac{k(n-1)}{n}}\left(a\left(\frac{k}{n}\right)_{n(n+1)}\right)=0$. Thus all terms in $(*)$ are zero, and consequently $a\left(s_{1}\right)_{12} \alpha^{s_{1}}\left(a\left(s_{2}\right)_{23}\right) \cdots$ $\alpha^{\sum_{t=1}^{n-1} s_{t}}\left(a\left(s_{n}\right)_{n(n+1)}\right)=0$ for any $k \in\{1,2, \ldots, m n-1\}$ and any choice of $s_{i}$ 's with $s_{1}+s_{2}+\cdots+s_{n}=k$.

Now recalling that $a\left(s_{1}\right)_{12} \alpha^{s_{1}}\left(a\left(s_{2}\right)_{23}\right) \cdots \alpha^{\sum_{t=1}^{n-1} s_{t}}\left(a\left(s_{n}\right)_{n(n+1)}\right)=0$ is equivalent to

$$
A_{s_{1}} \bar{\alpha}^{s_{1}}\left(A_{s_{2}}\right) \cdots \bar{\alpha}^{\sum_{t=1}^{n-1} s_{t}}\left(A_{s_{n}}\right)=0,
$$

we obtain $A_{s_{1}} \bar{\alpha}^{s_{1}}\left(A_{s_{2}}\right) \cdots \bar{\alpha}^{\sum_{t=1}^{n-1} s_{t}}\left(A_{s_{n}}\right)=0$ for any $k \in\{0,1,2, \ldots, m n\}$ and any choice of $s_{i}$ 's with $s_{1}+\cdots+s_{n}=k$. Therefore $U_{n+1}(R)$ is $\bar{\alpha}$-skew $n$-semi-Armendariz.

$(3) \Rightarrow(1)$ : Assume on the contrary that there is $0 \neq a \in R$ with $a \alpha(a)=0$. Let $A=\left(a_{i j}\right) \in N_{n}(R)$ with $a_{i(i+1)}=1$ for all $i$ and elsewhere zero, and $B=\left(b_{i j}\right) \in U_{n}(R)$ with $b_{11}=a, b_{n n}=-a$ and elsewhere zero. Then we have the following computation:

$$
\begin{array}{r}
A B \bar{\alpha}(A)=B \bar{\alpha}\left(A^{h}\right) B=B \bar{\alpha}(B)=0, A^{n-k} B=(-a) E_{k n}, B \bar{\alpha}\left(A^{k}\right) \\
=a E_{1(k+1)}
\end{array}
$$

for $k=1, \ldots, n-1$ and all $h$. Consider $f(x)=A+B x \in U_{n}(R)[x ; \bar{\alpha}]$. Then since $B \bar{\alpha}\left(A^{n-1}\right)=a E_{1 n}$ we have

$$
f(x)^{n}=\left(A^{n-1} B+B \bar{\alpha}\left(A^{n-1}\right)\right) x=\left((-a) E_{1 n}+a E_{1 n}\right) x=0
$$

by $(\dagger)$ but $A^{n-1} B, B \bar{\alpha}\left(A^{n-1}\right)$ are both nonzero. Thus $U_{n}(R)$ is not $\bar{\alpha}$ skew $n$-semi-Armendariz, a contradiction.

$(2) \Rightarrow(3)$ is obtained from Lemma $2(2)$ and the proofs of $(1) \Rightarrow(4)$, $(4) \Rightarrow(5)$, and $(5) \Rightarrow(1)$ are similar to the case of $U_{n}(R)$. 


\section{References}

[1] D.D. Anderson, V. Camillo, Armendariz rings and Gaussian rings, Comm. Algebra 26 (1998), 2265-2272.

[2] D.D. Anderson, V. Camillo, Semigroups and rings whose zero products commute, Comm. Algebra 27 (1999), 2847-2852.

[3] E.P. Armendariz, A note on extensions of Baer and P.P.-rings, J. Austral. Math. Soc. 18 (1974), 470-473.

[4] Y. Hirano, On annihilator ideals of a polynomial ring over a noncommutative ring, J. Pure Appl. Algebra 168, (2002), 45-52.

[5] C.Y. Hong, N.K. Kim, T.K. Kwak, On skew Armendariz rings, Comm. Algebra 31 (2003), 103-122.

[6] C.Y. Hong, N.K. Kim, T.K. Kwak, Ore extensions of Baer and p.p.-rings, J. Pure and Appl. Algebra 151 (2000), 215-226.

[7] C. Huh, Y. Lee, A. Smoktunowicz, Armendariz rings and semicommutative rings, Comm. Algebra 30 (2002), 751-761.

[8] Y.C. Jeon, Y. Lee, S.J. Ryu, A structure on coefficients of nilpotent polynomials, J. Korean Math. Soc. 47 (2010), 719-733.

[9] N.K. Kim, Y. Lee, Armendariz rings and reduced rings, J. Algebra 223 (2000), 477-488.

[10] J. Krempa, Some examples of reduced rings, Algebra Colloq. 3 (1996), 289-300.

[11] J. Lambek, On the representation of modules by sheaves of factor modules, Canad. Math. Bull. 14 (1971), 359-368.

[12] T.-K. Lee, T.-L. Wong, On Armendariz rings, Houston J. Math. 2 (2003), 583593.

[13] M.B. Rege, S. Chhawchharia, Armendariz rings, Proc. Japan Acad. Ser. A Math. Sci. 73 (1997), 14-17.

Department of Early Child Education

Kyungdong University

Kosung 219-830, Korea

E-mail: sbnam@k1.ac.kr

Department of Mathematics

Pusan National University

Pusan 609-735, Korea

E-mail: sjryu@pusan.ac.kr

Department of Mathematics

Pusan National University

Pusan 609-735, Korea

E-mail: pitt0202@hanmail.net 